\title{
Multi-objective Optimization of Demand Response in a Datacenter with
}

\section{Lithium-ion Battery Storage}

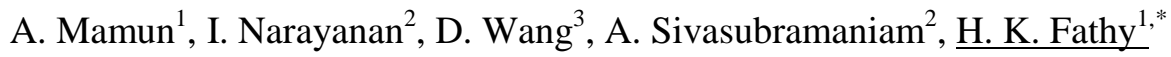 \\ ${ }^{1}$ Department of Mechanical and Nuclear Engineering \\ The Pennsylvania State University \\ University Park, Pennsylvania 16802, USA. \\ ${ }^{2}$ Department of Computer Science \& Engineering \\ The Pennsylvania State University \\ University Park, Pennsylvania 16802, USA. \\ ${ }^{3}$ Microsoft Research \\ One Microsoft Way \\ Redmond, WA 98052, USA. \\ *corresponding author. Phone: +1 8148674442 \\ e-mail: hkf2@engr.psu.edu
}

\begin{abstract}
This article optimizes lithium-ion battery management in a datacenter to: (i) maximize the dollar savings attainable through peak shaving, while (ii) minimizing battery degradation. To the best of the authors' knowledge, such multi-objective optimal datacenter battery management remains relatively unexplored. We solve this optimization problem using a second-order model of battery charge dynamics, coupled with a physics-based model of battery aging via solid electrolyte interphase (SEI) growth. Our optimization study focuses on a classical feedforward-feedback energy management policy, where feedforward control is used for peak shaving, and feedback is used for tracking a desired battery state of charge (SOC). Three feedforward-feedback architectures are examined: a proportional (P) control architecture, a proportionalintegral (PI) architecture, and a PI architecture with a deadband in its feedforward path. We optimize these architectures' parameters using differential evolution, for real datacenter power demand histories. Our results show a significant Pareto tradeoff between dollar savings and battery longevity for all architectures. The introduction of a deadband furnishes a more attractive Pareto front by allowing the feedforward controller to focus on shaving larger peaks. Moreover, the use of integral control improves the robustness of the feedback policy to demand uncertainties and battery pack sizing.
\end{abstract}


Keywords: Lithium-ion battery; Battery aging; Datacenters; Demand response; Optimization; Power management.

\section{Introduction}

This article examines the use of electrochemical batteries for datacenter demand response (DR). The article focuses on minimizing a Pareto combination of total electricity cost and battery aging by optimizing the control policy used for demand response. This work is motivated by the rapid growth of both the datacenter industry and its electricity needs. The cost of energy is a significant factor in datacenter operation. A large, energy efficient datacenter may, for instance, need as much as $10 \mathrm{MW}$ of electricity and spend approximately $\$ 1$ million on electricity bills per month, which is $30-50 \%$ of its monthly operating cost [1]. The high operating cost of purchasing electricity and the capital cost of the power infrastructure to draw electricity from the grid is affected by datacenter workload characteristics. Datacenter workloads often fluctuate significantly due to scheduled virus scans, media and cloud services, and flash crowd visitors $[2,3]$. Operating expenses $(\mathrm{OpEx})$ are affected by these fluctuations since utility companies often charge separately for peak power. Demanding a large amount of power during peak hours imposes additional cost penalties due to spot pricing or time-of-day tariffs [4]. A large capital expenditure (CapEx) is also required to enable the delivery of peak power through the power infrastructure, even though the probabilities of requiring peak power are often very low [5]. Figure 1 shows the normalized power demand for 7 days for one of the clusters in a Microsoft datacenter [6]. The figure shows a wide power demand range including a few large peaks and significant fluctuations in cluster-level power demand. 


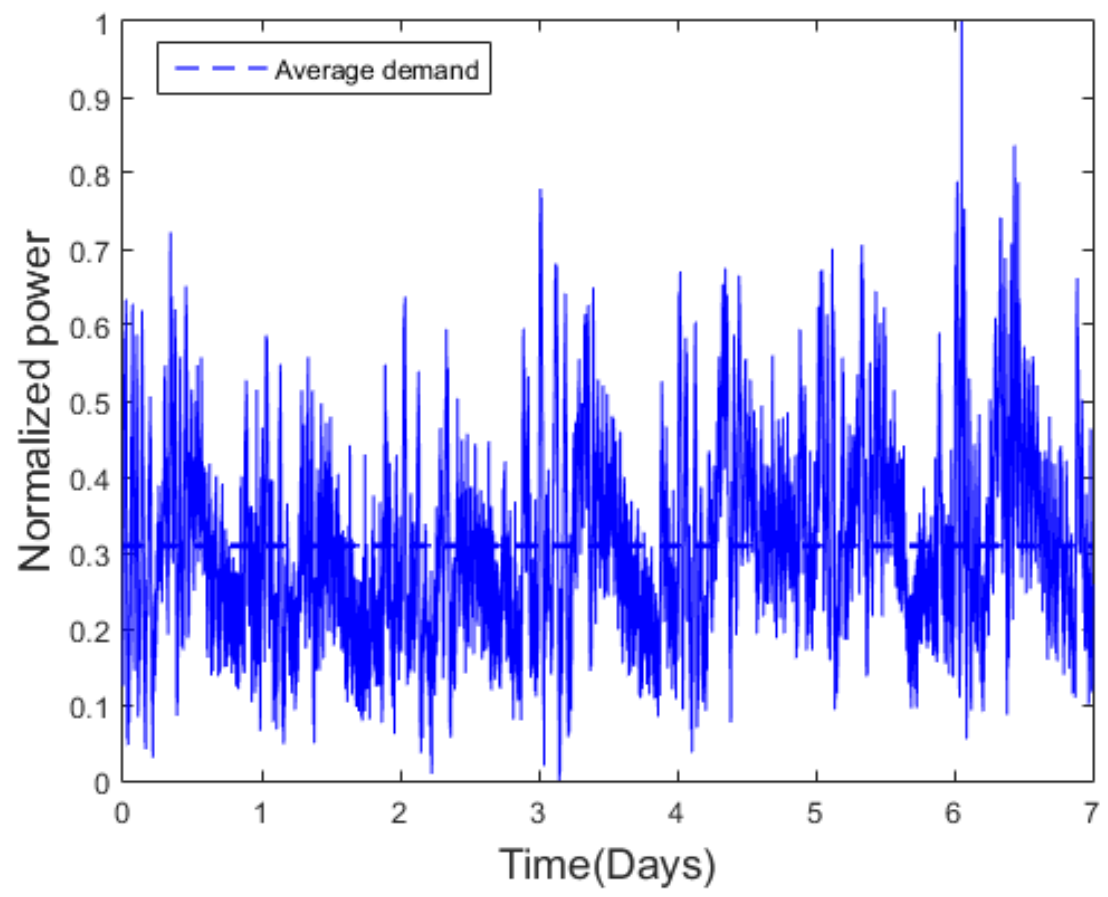

Fig. 1. Cluster level normalized power demand for a Microsoft datacenter. (Single column fitting image)

The economic penalty associated with fluctuating datacenter power demand can be reduced through demand response. The term "demand response" refers to any process that changes consumer electricity demand based on electricity price [1]. Researcher have considered different information theoretic (IT) [710] and cooling capacity knobs [11-13] to perform demand response in datacenters. The IT knobs are used to (i) "throttle" workload by reducing server speeds when electricity is expensive, (ii) "shift" workload temporally from peaks to adjacent valleys, or (iii) "transfer" the workload to other datacenters experiencing either smaller workloads or cheaper energy availability (or both). Since these knobs involve tradeoffs between energy cost and performance degradation, energy storage has come into play as an additional knob. The idea is to store grid electricity when it is either abundant or inexpensive (or both) and use the stored energy during high workload or peak hours.

The literature already examines the problem of utilizing existing storage in datacenter uninterruptible power supply (UPS) systems or using a separate battery pack for demand response $[1,4,5,14]$. The 
primary research focus of demand response in datacenters with UPS is the minimization of total cost of ownership (TCO) $[15,16]$. This is the sum of amortized capital and operating costs over a time horizon [17]. Queuing theory-based Lyapunov optimization techniques are also used to find near-optimal solution to minimize monthly electricity bills $[1,18]$. These optimization analyses typically focus on lead-acid battery storage, although lithium-ion batteries are also considered by some researchers. For demand response optimization, lithium-ion batteries are generally modeled purely as charge integrators, and battery lifetime is modeled using depth of discharge (DOD) based lifetime charts and/or charge processed models [16,19-21]. These models are quite limited in their ability to capture the fundamental physical phenomenon affecting lithium-ion battery behavior. For example, an elementary charge integration model does not capture internal battery diffusion dynamics, and therefore fails in capturing the dynamic constraints imposed on battery charging/ discharging by diffusion. One goal in this article is to extend the existing literature on datacenter demand response through the use of a lithium-ion battery model that captures both charge integration and voltage relaxation dynamics.

In addition to using a suitable battery model, a control scheme is also necessary to optimally utilize batteries for demand response. Optimal battery utilization in this case means using the batteries to minimize the electricity cost as much as possible with minimum battery degradation. Load leveling using batteries will reduce the discounted monthly capital and operating cost associated with datacenter electricity demand. However, deep charge and discharge to shave large peaks might accelerate battery aging. Therefore using battery charge capacity for load leveling during demand response becomes a multi-objective optimization problem, where minimizing battery health degradation and electricity costs are the two competing objectives. To the best of our knowledge, the extensive study of control strategies for model-based, health-aware battery control during demand response remains relatively unexplored in the datacenter power management literature. Previous work by authors addresses this issue by designing an optimal proportional controller for Li-ion batteries in large-scale datacenters [22]. The work assumes the availability of an optimally sized, power-efficient storage system (i.e., flywheels or ultracapacitors) 
for emergency power plus a separate Lithium-ion battery pack for demand response since such a hybrid storage solution is often more cost-effective than a battery-only solution [23]. In this previous work we (i) build a second-order model of a Lithium-ion battery that captures both ohmic and diffusion dynamics, (ii) use an empirical capacity fade model to capture battery aging, (iii) design a proportional feedforwardfeedback controller for demand response, and (iv) find the optimal gains and parameters of the controller via differential evolution. This article builds on the above work and extends it significantly in the following ways. First, instead of using an empirical capacity fade model, this paper builds a physics-based model of lithium-ion battery aging via solid electrolyte interphase (SEI) layer growth. This changes the optimization objective pertaining to battery aging from collective capacity fade to a more fundamental metric, namely, the loss of cyclable lithium due to SEI layer growth. Second, unlike the above previous work, we do not limit the battery control policy to be governed solely by a classical proportional feedforward/feedback controller. Rather, we explore the augmentation of this proportional controller's feedforward path with a deadband. This is a standard tool from the control system design domains that causes the controller to focus solely on shaving large demand peaks, thereby reducing unnecessary utilization during smaller peaks. We also explore the augmentation of the above control law with an integral controller in the feedback loop. Integral feedback guarantees the robust tracking of a desired battery state of charge even in the presence of certain modeling uncertainties. For example, if the datacenter's average electricity demand changes unexpectedly, or if datacenter battery storage capacity changes (due to aging), integral control will compensate for these effects and continue to track desired battery state of charge levels. Third, sizing analysis is performed to understand the impact of battery pack size on the above competing optimization objectives.

The remainder of the article is organized as follows. The article presents (i) a second order Lithiumion battery model with a nonlinear output equation; (ii) a physics-based SEI layer growth model; (iii) feedforward-feedback control policies for optimal demand response; and (iv) the formulation and solution 
of the above multi-objective optimization problem. The article then concludes by describing the results and summarizing the lessons learned from the study.

\section{Lithium-ion battery model}

The Lithium-ion battery model used in this study is a second-order equivalent-circuit (EC) model shown in Figure 2. The optimization framework presented here can be extended to incorporate more complex battery models. The EC model captures some of the important battery dynamics through simple electrical component representation and is also suitable for control purposes. The open-circuit voltage $(\mathrm{OCV})$ of the cell is a nonlinear function of state of charge (SOC) and represented by a voltage source $\left(\mathrm{V}_{\mathrm{OC}}\right)$ in Figure 2. The series resistance $\mathrm{R}$ represents the collective resistance of the cell and cell holder. The parallel resistance-capacitance $(\mathrm{RC})$ pair represents the voltage relaxation due to transient effects, especially diffusion polarization effects. The terminal cell voltage and input current are denoted by V(t) and I(t), respectively. The nonlinear OCV-SOC curve, resistances and capacitance of the circuit representation are obtained from experiments described in [24]. In these experiments, a set of Lithium iron phosphate $\left(\mathrm{LiFePO}_{4}\right) 18650$ cells are tested using a commercial cycler. The equivalent circuit model parameters used in this paper are listed in Table 1.

Table 1. Equivalent circuit model parameters for Lithium-ion 18650 cell. (Single column fitting table)

\begin{tabular}{ll}
\hline Parameter & Value \\
\hline Cell capacity, $Q(\mathrm{C})$ & $3.9447 \times 10^{3}$ \\
Resistance, $R(\Omega)$ & 0.0285 \\
Capacitance, $C_{r}(\mathrm{~F})$ & $2.0523 \times 10^{4}$ \\
Time constant, $\tau=C_{r} R_{r}(\mathrm{~s})$ & $3.4997 \times 10^{3}$ \\
\hline
\end{tabular}




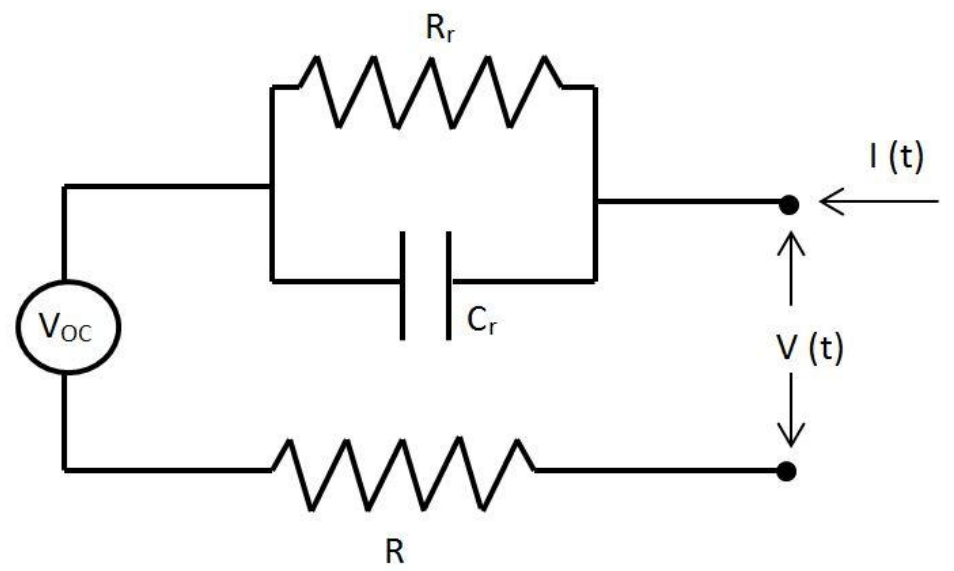

Fig. 2. Equivalent circuit model of a Lithium-ion battery. (Single column fitting image)

The state-space representation of the equivalent circuit model is the described in Equation (1)-(3).

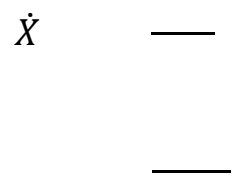

Here $X_{1}(t)$ is the state of charge (SOC) of the battery, $X t$ ) is the charge in the capacitor, $V_{O C}$ is the open-circuit voltage which is a function of $X_{1}(t)$, and $I($ and $V(t)$ are the input current and output (terminal) voltage, respectively. For a given input power $t$ ), the corresponding current can be found from the expressions in Equation (4) and (5). 


\section{Battery health degradation model}

In this section we built a physics-based static degradation map to quantify the capacity fade of the lithium-ion battery used for demand response. The map is built based on the solid electrolyte interphase (SEI) layer growth model proposed by Ramadass et al. [25]. The initial formation of the SEI layer due to side reactions is crucial for proper cell operation. However, continuous solvent reduction and deposition of reaction product increase the thickness and resistance of the SEI layer in the negative electrode. Consumption of active Lithium in this side reaction results in irreversible loss in cell charge capacity. The irreversible capacity loss due to side reaction in the negative electrode can be expressed by Equation (6).

$$
\frac{\partial Q_{\text {loss }}(t)}{\partial t}=-\int_{0}^{L_{n}} J_{\text {side }}(t) A_{n} d x
$$

Here $Q_{\text {loss }}(t)$ is the time-varying capacity loss due to side reaction which is responsible for SEI layer growth. The local volumetric current density for this side reaction $\left(J_{\text {side }}\right)$ is represented by the Tafel equation in Equation (7).

$$
J_{\text {side }}(t)=-i_{0, s} a_{n} \exp \left(\frac{-0.5 F}{R_{u} T} \eta_{s}(t)\right)
$$

Here $i_{0, s}, \eta_{s}, R_{u}, a_{n}, F$, and $T$ represent the exchange current density for the side reaction, side reaction over potential, universal gas constant, specific surface area, Farady's constant, and cell temperature, respectively. The side reaction overpotential $\left(\eta_{s}\right)$ can be expressed using Kirchhoff's voltage law in Equation (8).

$$
\eta_{s}(t)=\Delta \varphi(t)-U_{s, r e f}-\frac{J_{t o t}(t)}{a_{n}} R_{f i l m}(t)
$$

Here $\Delta \varphi$ represents the potential difference between the solid and the electrolyte, and $U_{s, r e f}$ represents the equilibrium potential of the solvent reduction reaction. The total volumetric current density in the negative electrode $\left(J_{\text {tot }}=I /\left(A_{n} L_{n}\right)\right)$ is the sum of main reaction current density $\left(J_{\text {main }}\right)$ and side reaction current density $\left(J_{\text {side }}\right)$ expressed in Equation (9).

$$
J_{\text {tot }}=J_{\text {main }}+J_{\text {side }}
$$


In Equation (8), represents the resistance of the SEI film. It is assumed to grow in proportion to its thickness and add to the initial resistance of SEI layer $\left(R_{S E I}\right)$ given in Equation (10).

Here, is the time varying film thickness and is the conductivity of the film. The growth rate of film thickness is governed by (11).

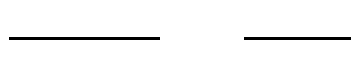

Here, $M_{P}$ and $\rho_{P}$ represent molecular weight and mass density of the reaction product, respectively. The main reaction overpotential of the negative electrode $\left(\eta_{n}\right)$ can be expressed similar to Equation (8) using Kirchhoff's law in Equation (12).

Substituting from Equation (12) into Equation (8) results in a simplified form given in Equation (13).

Since the side reaction exchange current density is orders of magnitude smaller than the main reaction current density, we can assume $J_{\text {tot }} \sim J_{\text {main }}$ in Equation (9). With this assumption, the main reaction overpotential of the negative electrode $\left(\eta_{n}\right)$ in Equation (13) can be found by solving the Butler-Volmer equation given in Equation (14).

Here $\alpha_{a}$ and $\alpha_{c}$ are the apparent charge transfer coefficients for anode and cathode and $i_{0, n}$ represents the main reaction exchange current density at the negative electrode. The main reaction exchange current density $\left(i_{0, n}\right)$ at the interface of the negative electrode can be found using Equation (15). 
Here $C_{s, n, \max }$ and $C_{s, n, s u r f}$ represent the maximum concentration and the surface concentration of Lithium-ion in the negative electrode. The concentration of Lithium-ion in the electrolyte is denoted by $C_{e}$. The surface state of charge $\left(x_{n, \text { surf }}\right)$ for the negative electrode can be defined as the ratio of surface ion concentration and maximum ion concentration in the negative electrode mentioned in Equation (16).

For simplicity in control design, given the surface SOC at the negative electrode and input current, we construct a static map of capacity fade from the above model described in Equation (6) - (16). To construct the map, we assume spatially averaged instantaneous film growth similar to [26]. The physical cell parameters and the SEI layer parameters (Table 2) are obtained from [27] and [28], respectively. The map is constructed by calculating instantaneous capacity loss rate corresponding to different combinations of possible input current and surface SOC of the negative electrode. Figure 3 presents the static degradation map where large charging currents at high SOCs accelerate the capacity loss rate.

One complication in using the above capacity fade model is that the EC model representing the lithium-ion cell only has a bulk SOC state variable to represent the charge in the cell. Therefore, to find the capacity fade of the lithium-ion battery represented by the EC model we have assumed the bulk cell SOC $\left(X_{1}(t)\right)$ from Equation (1) to be equal to the surface SOC of the negative electrode $\left(x_{n, \text { surf }}\right)$ in Equation (16). This is a reasonable assumption since the diffusion dynamics of the electrolyte and the positive electrode are much faster compared to the negative electrode [29]. Based on this assumption, the capacity fade rate is found from the map at every simulation time step given the input current and the cell SOC from the EC model. The capacity fade rate is integrated over the simulation time length and the total loss in charge capacity is used as the measure of health degradation in demand response optimization. 
Table 2. SEI layer model parameters for Lithium-ion 18650 cell. (Single column fitting table)

\begin{tabular}{|c|c|}
\hline Parameter & Value \\
\hline Side reaction equilibrium potential, $U_{s} \quad f(\mathrm{~V})$ & 0.4 \\
\hline Side reaction exchange current density, $\quad\left(\mathrm{A} \mathrm{m}^{-2}\right)$ & $3.9 \mathrm{e}-8$ \\
\hline SEI layer molar mass, $M_{p}\left(\mathrm{~kg} \mathrm{~mol}^{-1}\right)$ & 0.162 \\
\hline SEI layer density, $\rho_{p}\left(\mathrm{~kg} \mathrm{~m}^{-3}\right)$ & 1690 \\
\hline
\end{tabular}

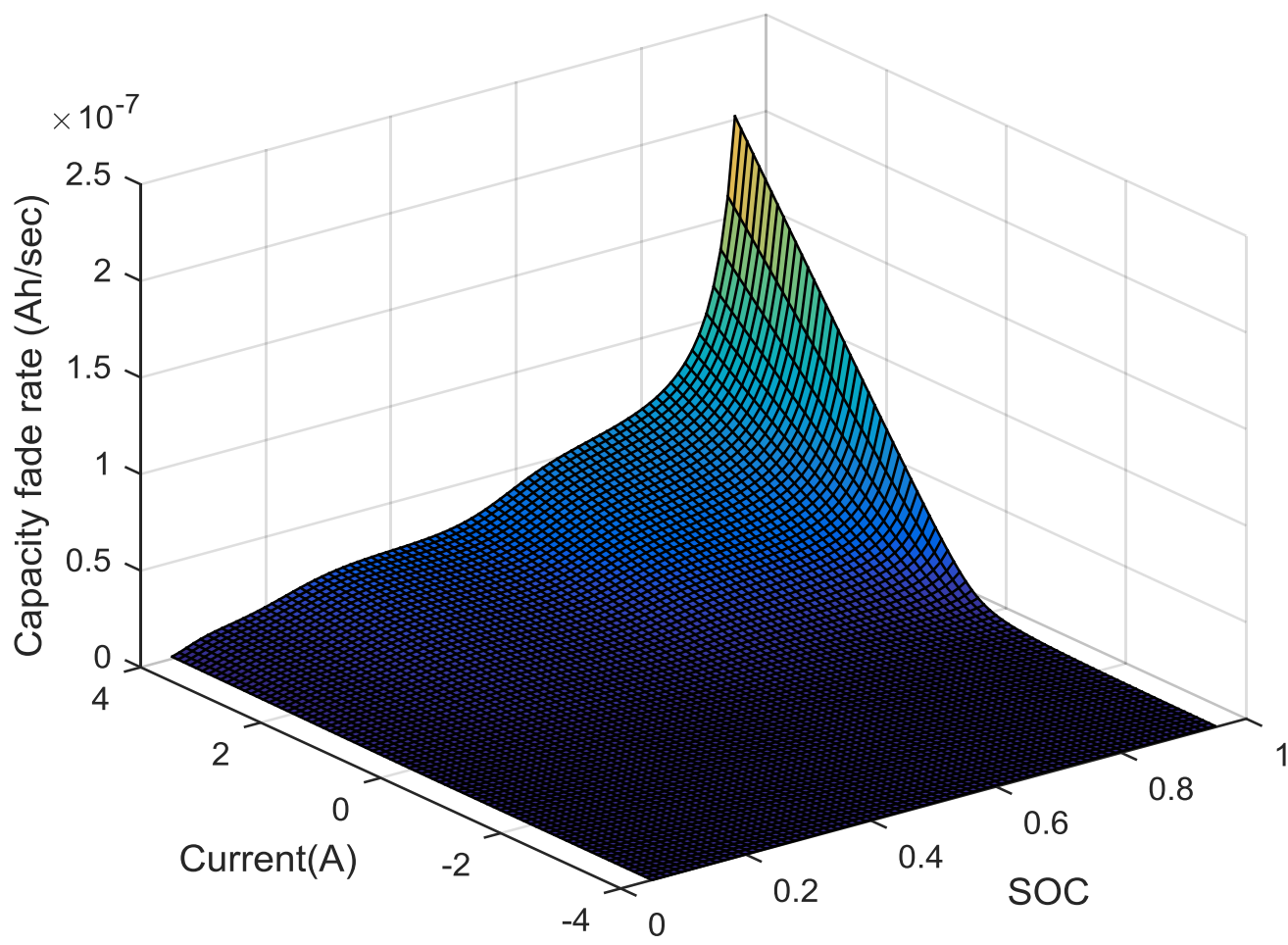

Fig. 3. Surface plot of battery health degradation. (Single column fitting image)

\section{Savings in datacenter capital and operating expenses}

The economic benefits of demand response using batteries can be expressed in terms of savings in capital and operating expenses. The datacenter power infrastructure and delivery networks are typically overprovisioned to accommodate peak power draws for safety, even though the peaks are rare [8]. Capital expenditure $\left(C_{c a p}\right)$ for provisioning the required power capacity for a typical datacenter is estimated to be 
$\$ 10-20 /$ Watt assuming a12-year service life $[4,19]$. Therefore, if batteries are used for demand response to reduce peak power draw from utilities, each watt of peak power reduction will lead to $\$ 10-20$ savings over the datacenter lifetime. In this article a conservative capital savings of $\$ 10 /$ Watt is assumed. Using the straight-line method of depreciation, monthly savings in capital expense (CapEx) are computed in Equation (17). Here $P_{c a p, \max }$ and $P_{d, \max }$ are the peak power levels demanded from utilities with and without demand response, respectively.

Utilities usually penalize high power draw from grid through peak-based pricing and time-of-day pricing during peak hours. Therefore, monthly operating expenses are also reduced when batteries are used to shave large peaks. In this work, peak-based pricing is considered where utilities impose a monthly “demand charge" per kW when power draw exceed a predefined limit [30]. However, peaks are penalized equally throughout the day irrespective of the time of occurrence. Equation (18) shows OpEx savings coming from reduction in demand charge and change in total energy consumption per month. The first term indicates savings due to peak shaving and the second term indicates savings due to energy discharged from the Lithium-ion battery pack. The amount of energy stored and released from the cell at time step $i$ is denoted by $E_{\text {discharge, } i}$ and $E_{\text {charge, } i}$, respectively. Since total energy is conserved, the change in net energy comes from the difference in initial and final states of charge in the lithium-ion battery pack. Typical energy price per $\mathrm{kWh}\left(C_{\text {energy }}\right)$ and the penalty for each $\mathrm{kW}$ peak power above predefined limit $\left(C_{\text {peak }}\right)$ are obtained from [19] and listed in Table 3. 
Table 3. Capital and operating expenses related to electricity consumption in datacenters. (Single column fitting table)

\begin{tabular}{lll}
\hline Parameters & & Value \\
\hline Capital cost of power provisioning, $\quad\left(\$ \mathrm{~W}^{-1}(12 \text { years })^{-1}\right)$ & 10 \\
Unit cost of energy, $\quad\left(\$(\mathrm{kWh})^{-1}\right)$ & 0.05 \\
Unit cost of peak power, $\quad\left(\$ \mathrm{~kW}^{-1}\right.$ month $\left.^{-1}\right)$ & 12 \\
\hline
\end{tabular}

\section{Control policy for demand response using batteries}

To use a battery pack for demand response, first a control policy is necessary to decide when and how much to charge or discharge the batteries. During demand response, we want to reduce variations in power drawn from utilities as much as possible. For "ideal" load leveling, a constant power is drawn from utilities and any fluctuations are compensated from the battery pack. Here, this constant power is the temporal average of power demand profile which is defined as the desired power cap $\left(P_{c a p, \text { des }}\right)$. Given datacenter power demand $\left(P_{d}(t)\right)$ at time $\mathrm{t}$, the battery power $\left(P_{b a t t}(t)\right)$ required to achieve "ideal" load leveling is given in Equation (19).

When $P_{d}(t)$ is higher than $P_{c a p, d e s}$, the battery pack needs to discharge to provide the rest of the power to run the datacenter. Therefore, sign of $P_{b a t t}(t)$ is opposite of the sign of $P_{d}(t)$ in Equation (19). However, it is conceivable that such "ideal" load leveling could exceed battery power and energy limitations. This problem is a consequence of the fact that Equation (19) is a "feedforward" control law: it operates the datacenter batteries for demand response, but does not adjust the operation to account for battery limitations.

In addition to the above feedforward control law for load leveling, a feedback law is needed in the overall control policy to track a desired SOC and avoid hitting SOC bounds. Tracking a desired cell SOC is also important to control battery aging, since battery aging depends strongly on SOC according to the 
aging model used in this article. Therefore, our proposed controller is a combined feedforward-feedback controller. Feedforward-feedback controller is one of the most effective ways to achieve multiple goals such as tracking a reference and at the same time making sure that the state of the underlying system does not significantly deviate from its desired range. The simplest feedforward-feedback controller is a proportional $(\mathrm{P})$ feedforward-feedback controller which can be used to determine a battery power level while performing load leveling and SOC tracking at the same time. Figure 4.a shows the block diagram of the P controller where the feedforward component in Equation (19) is multiplied by a gain $\alpha$ to allow partial load leveling and a feedback component is added with a gain $\beta$ for SOC tracking. The control policy is shown in Equation (20) where controller decides on battery power $P_{\text {batt }}(t)$ given the datacenter power demand $\left(P_{d}\right)$ and battery state of charge $(S O C)$ at time t.

In Equation (20), $\alpha$ and $\beta$ are positive proportionality constants and $S O C_{d e s}$ is the desired SOC to track. The optimal values of these proportionality constants and the desired SOC depend on multiple factors such as physical cell limits, expected battery aging, and electricity cost. In this paper, we find these optimal and non-trivial (other than $\alpha=1$ and $\beta=0$ ) controller gains and parameters by solving a multiobjective optimization problem.

The proportional feedback of the above control law can only minimize the steady state error in SOC tracking but cannot eliminate it. In contrast, proportional-integral control is one of the most standard approaches in feedback control for tracking a desired reference by eliminating steady state error. Therefore, to obtain zero steady-state error in tracking the desired SOC, an integral component can be added in the feedback loop of the proportional feedforward-feedback controller. Equation (21) shows the resulting proportional-integral (PI) controller which has one additional unknown parameter to be found for optimal demand response, namely, $\delta$, the integral gain of the controller. 
In the above two controllers, the power drawn from the battery pack varies linearly with the datacenter power demand irrespective of the magnitude of the demand. However, shaving smaller demand peaks does not contribute to electricity cost savings as much as shaving large peaks. Therefore, it might be possible to find a better demand response strategy when the controller uses the battery power only when power demand exceeds a certain limit. Adding a deadband in the feedforward component of the control law can allow the controller to implement this idea without affecting the tracking capability of the feedback part. Figure 4.b shows an illustration of the feedforward component of the control law with a deadband. The parameter $\gamma$ determines the width of the deadband. If $P_{d, \max }$ and $P_{d, \min }$ are maximum and minimum power demand, respectively, then $\gamma\left(\left|P_{d, \max }\right|+\left|P_{d, \min }\right|\right)$ is the width of the deadband. Within this deadband, the feedforward component in Equation (21) is zero, which means that the controller does not react to demand peaks and acts as a PI feedback controller for tracking desired SOC only. Outside the deadband, battery power varies linearly with power demand similar to the P and PI controller. For the PI controller with deadband, the width of the deadband $(\gamma)$ is defined as the fifth optimization variable in addition to the four unknown parameters $\alpha, \beta, \delta$, and $S O C_{d e s}$. Therefore, for this variation of control law, the optimization algorithm searches for an optimal deadband in conjunction with other unknown parameters to achieve minimum electricity cost and battery health degradation during demand response. 


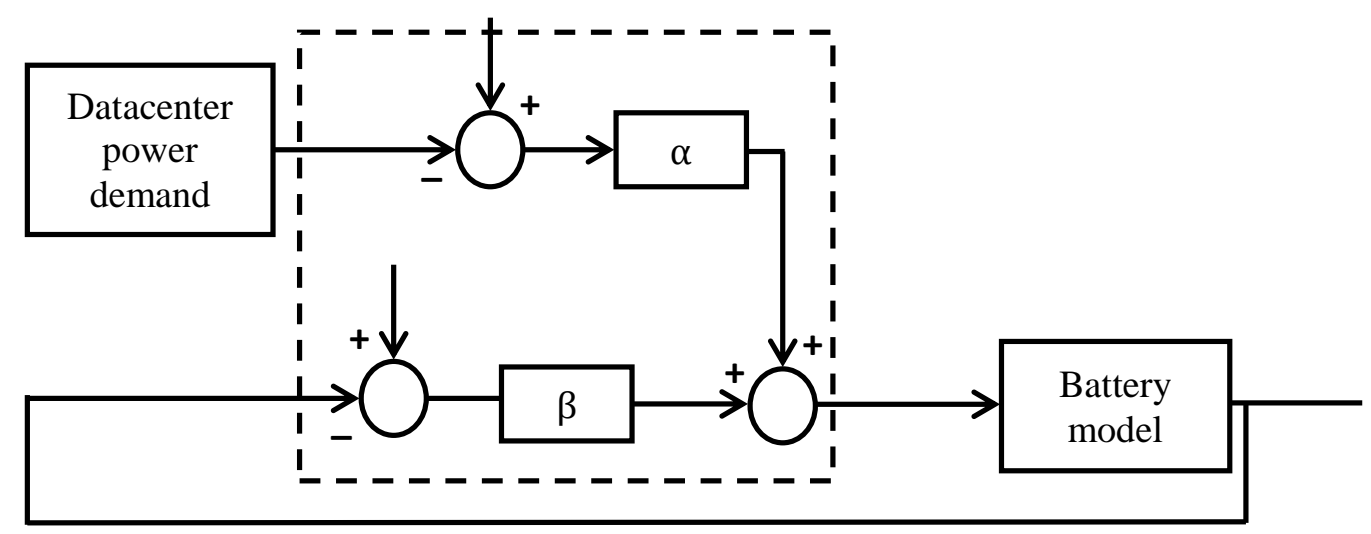

(a)

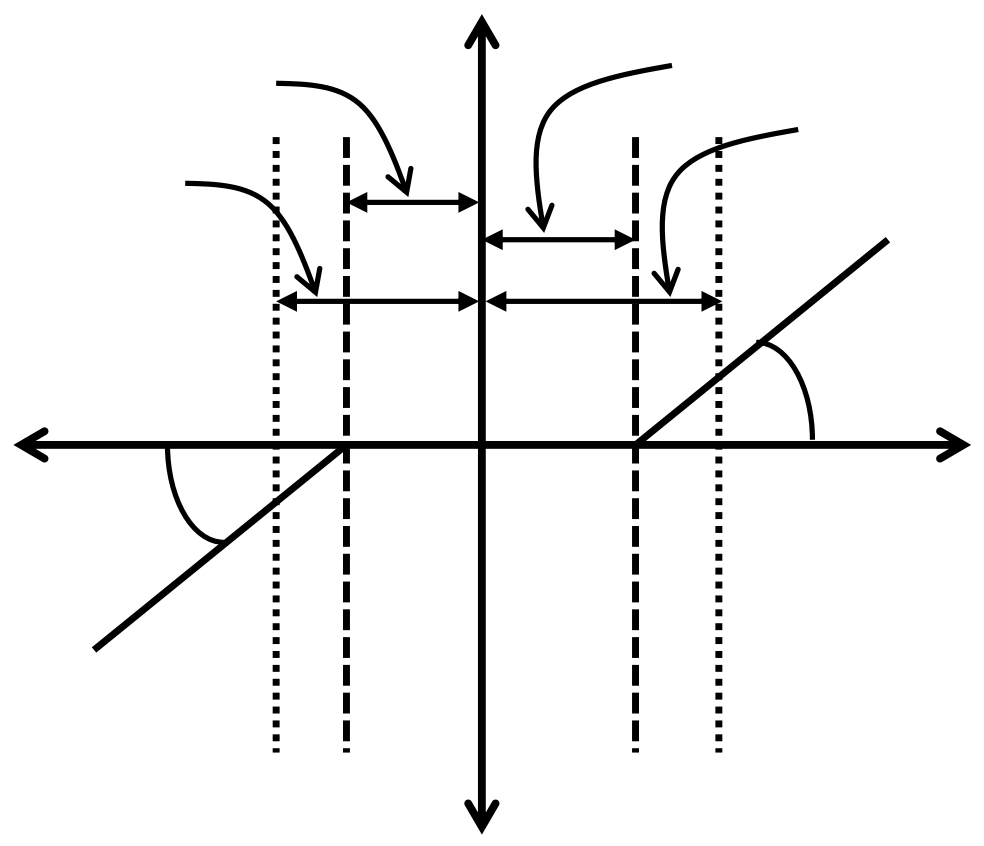

(b)

Fig. 4. a) Block diagram of battery control system with proportional feedforward-feedback controller. ; b) Feedforward part of PI controller with deadband. (Single column fitting image)

\section{Multi-objective optimization for demand response}

\subsection{Optimization problem formulation}

The ability of the above controllers to achieve one or more of the optimization objectives depend on the unknown parameters: $\alpha, \beta, \delta, \gamma$ and $S O C_{\text {des. }}$ A multi-objective optimization problem is formulated and solved to find the optimal values of these unknown parameters for each type of controller. In this work, 
we have two optimization objectives to perform demand response with batteries: (i) maximize savings in datacenter capital and operating expenses and (ii) minimize capacity fade due to SEI layer growth. Intuitively, these two objectives are competing in nature. High peak shaving will result in more dollar savings, but intense usage might cause higher battery health degradation. Moreover, the intended peak shaving might not be possible due to physical battery limitations such as voltage and SOC limits. Initial cell SOC is also an unknown parameter. To avoid additional complexity, we assume that cell SOC at $\mathrm{t}=0$ equals the desired SOC. Therefore, the goal of the multi-objective optimization problem is to find a set of values of each unknown parameter for each controller so that both optimization objectives are attained.

To achieve this goal, an evolutionary algorithm is used to generate a Pareto front of the two objectives. It may be possible to associate a dollar value with degraded battery capacity and lump the two objectives into a single cost function. However, the weighting needed for such lumping of objectives is not known, hence the explicit multi-objective approach is adopted here and presented in Equation (22). A negative sign is added in front of the second objective to solve the multi-objective optimization problem as a minimization problem. In Equation (22), dt represents the simulation time step which is 20 seconds in this work. The CapEx and OpEx savings rate (\$/dt) given in Equation (22) are computed by dividing the CapEx and OpEx savings rate (\$/day) given in Equation (17) and (18) by the number of simulation time steps in a day.

\section{Subject to}

Battery SOC constraints: 5

Battery terminal voltage constraints: 2

Maximum discharge power constraint: 
Controller parameter constraints:

The first two constraints of the optimization problem ensure safe operation of the Lithium-ion cell. The third constraint limits the maximum discharge power for simulation stability. The remaining constraints define the allowable design space of the optimization variables. For example, the non-zero minimum value of $\alpha$ ensures that for any kind of control strategy, a portion of the battery capacity will always be used for demand response.

\subsection{Solution method using modified differential evolution}

To solve the above multi-objective optimization problem, differential evolution is used. This is a population-based evolutionary algorithm first proposed by Storn and Price [31]. This article takes the standard differential evolution algorithm and incorporates an additional mutation operator (namely, a random walk imposed on population members) to create additional sets of candidate solutions. To optimize datacenter demand response using batteries, the differential evolution algorithm is combined with datacenter workload, the controller, the battery charge-discharge model, and the health degradation model. The goal is to find a set of optimal control parameters for each controller design which generate a Pareto front of the two competing objectives. An overview of the optimization process is given in Table 4. 
Table 4. Differential evolution algorithm for optimal datacenter demand response. (Single column fitting table)

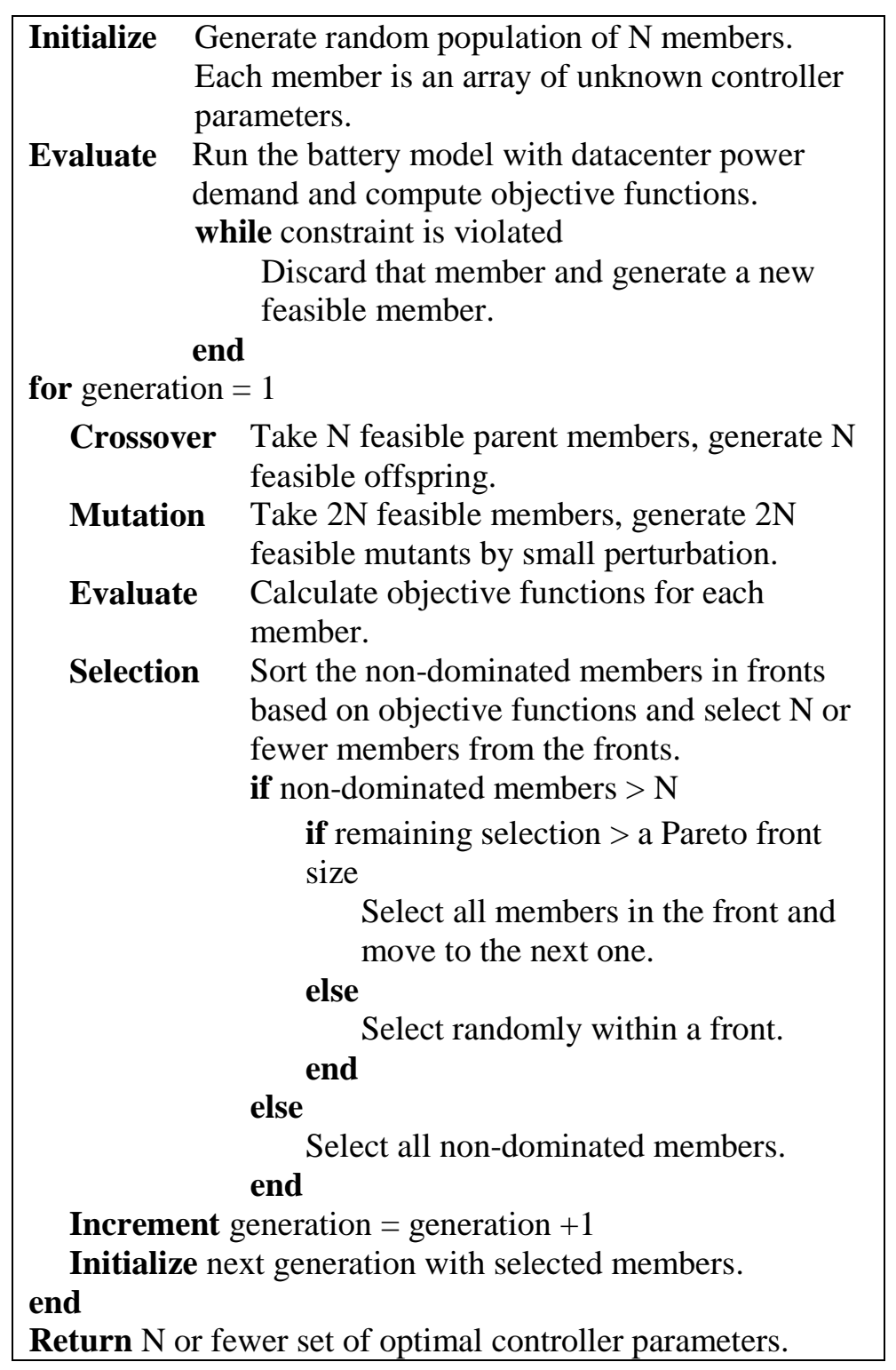

\section{Simulation results}


This section presents the Pareto optimal solution set for the optimization problem presented in the previous section for each controller presented in section 5. A Pareto front is obtained for each controller and every point in the Pareto front corresponds to a particular set of optimal controller parameters. In this section, the shape of the Pareto fronts and change in controller parameters along the fronts are examined. The optimal controller designs are then used for demand response simulation to study their load leveling capability and robustness. The Pareto tradeoff for different battery pack sizes is also examined to understand the impact of sizing on optimization objectives.

\subsection{Generation of demand response profile}

The first step for datacenter demand response optimization is to generate a demand response trajectory from the power demand. The datacenter power demand profile used in this article comes from a cluster of racks of servers in a Microsoft datacenter [6]. To perform demand response, each cluster is assumed to contain a Lithium-ion battery pack with 1000 cells of 1.1 Ah charge capacity. We are interested in minimizing fluctuations in power demand. Therefore, the temporal average of the power demand profile $\left(P_{d, a v g}\right)$ is subtracted from the original profile and a zero mean demand response (DR) profile is generated. This DR profile is then scaled for a single Li-ion cell assuming the availability of 1000 cells in the cluster level battery pack and shown in Figure 5. The controllers' job is to flatten this demand response profile as much as possible using battery power and energy capacity. The following two assumptions are made in this regard: (i) constant power draw equal to $P_{d, a v g}$ is always available from grid without any peaking penalties, and (ii) the datacenter power infrastructure and distribution network are already provisioned to handle $P_{d, a v g}$. 


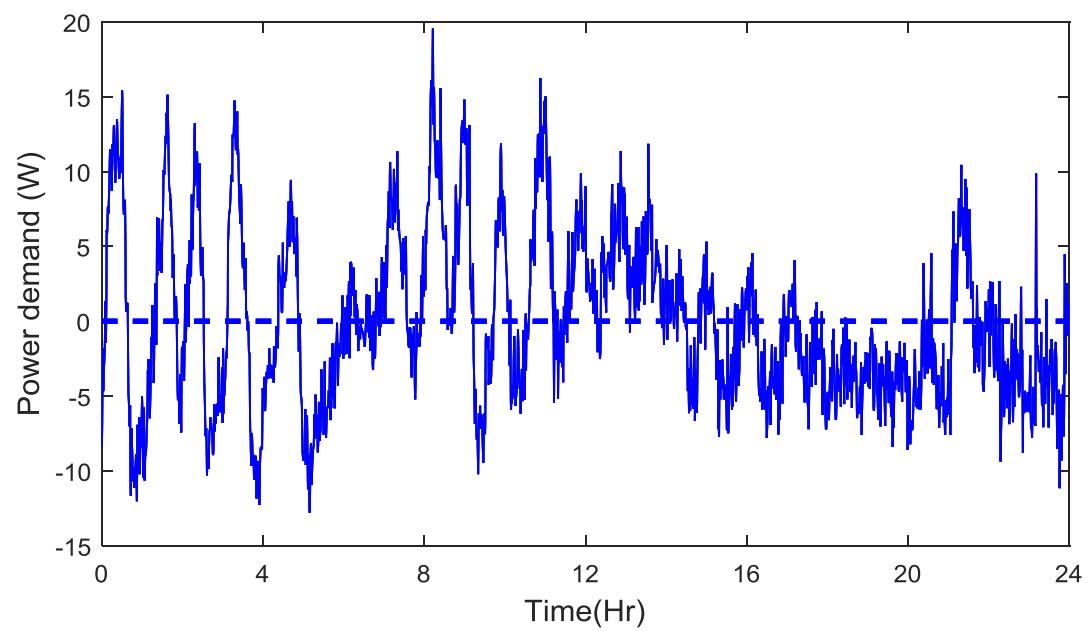

Fig. 5. Datacenter demand response profile scaled for a single cell in a Li-ion battery pack with 1000 cells. (Single column fitting image)

\subsection{Pareto fronts and controller parameters for P controller}

Given the above demand response profile, this section presents the solution of the multi-objective optimization problem using population-based differential evolution algorithm. First, the Pareto optimal solutions are found for the proportional feedforward-feedback controller (Equation (20)) and the resulting Pareto front is shown in Figure 6.a. All the solutions in this Pareto front are "optimal", in the sense that neither of the objective functions can be improved without penalizing the other. The objective function value corresponding to each optimal set of $\alpha, \beta, S O C_{d e s} \delta$ and $\gamma$ represents one point in the Pareto front. The P controller is a subset of the PI controller and the PI controller with deadband. Therefore, the optimal solutions of the P controller are also feasible for the PI controller and the PI with deadband. To speed up the convergence, $20 \%$ of the optimal solutions for the $\mathrm{P}$ controller are used as the initial population for optimizing the parameters of the PI controller and PI controller with deadband. The remaining $80 \%$ of the initial population is randomly generated to maintain population diversity. The Pareto fronts generated from the solution of the optimization problems for PI controller and PI controller with deadband are also shown in Figure 6.a. In the leftmost point of a Pareto front the controller puts maximum emphasis on minimizing degradation and on the rightmost point controller puts maximum emphasis on dollar savings. From left to right, the savings increases nearly 10 times while the degradation 
rate also increases nearly 6 times. Therefore these two objectives have significant design tradeoffs. Figure 6.a also shows that the Pareto front from the PI controller stays very close to the Pareto front from P controller with very small improvements. In contrast, the PI controller with deadband shows significant improvements in the objective functions compared to the other two.

The slope of the Pareto fronts in Fig. 6.a, particularly for the PI controller with deadband, undergoes a sharp transition as one traverses this front. Below this transition, one can improve cost savings significantly with minimal increases in battery degradation. Above this transition, one sees diminishing returns, in the sense that additional dollar savings are only attainable with significant degradation penalties. This phenomenon can be explained as follows: the datacenter batteries can achieve relatively small dollar savings with ease, simply by shaving infrequent and narrow peak power demand events. This does not exercise the batteries excessively, and therefore does not translate to an excessive increase in degradation. However, to achieve greater savings, one eventually needs to shave excess power demand for longer time durations. This taxes the datacenter batteries more heavily, thereby accelerating degradation significantly and causing the phenomenon of diminishing returns.

The shape of the Pareto front i.e., the position of the Pareto points in the objective function space can be understood from the corresponding optimal controller parameters in the variable space. Figure 6.b to 6.f show the optimal values of controller gains and parameters at each point in the Pareto front. For the $\mathrm{P}$ controller, Figure 6.b shows increasing value of $\alpha$ as one traverse the Pareto front from left to right. For larger $\alpha$ values, the controller puts more emphasis on peak height and tries to shave the peak as much as possible. To shave large peaks, deep charge and discharge of Li-ion cell is necessary. The controller allows deep charge-discharge by putting less emphasis on tracking the desired SOC either by lowering $\beta$ or shifting $S O C_{d e s}$ towards the middle of the SOC range. Figure 6.c shows the change in $\beta$ where it decreases at the beginning but varies in small amount for the rest of the Pareto points. Figure 6.d shows an increasing trend in $S O C_{d e s}$. These two figures indicate that average battery SOC has a non-trivial impact on the optimization objectives. 
Every Pareto-optimal control design in Fig. 6.a can be examined from a cost-vs.-benefit perspective as follows: one can divide the dollar savings attained per cluster per day by the corresponding SEI layer growth-related capacity fade. In doing so, noting that there are 1000 battery cells per cluster in this study, one obtains a 'slope' with units of dollar savings per Ampere-hour of SEI growth-related capacity fade. Figure 6.a shows three lines representing different values of this 'slope'. By traversing the Pareto front, one can make informed decisions regarding the relative importance of dollar savings, battery health, and/or the cost-benefit metric of savings per unit health degradation. The precise choice of a Paretooptimal control policy will depend on factors such as the initial deployment cost of the datacenter batteries and the degree to which SEI layer growth contributes to overall battery degradation. Consider, for example, an over-simplified scenario where the initial battery deployment cost is $\$ 250$ per Amperehour, where $20 \%$ capacity fade translates to battery death, and where SEI layer growth is responsible for all capacity fade. In such a scenario, any control policy whose savings are guaranteed to have a net present value (with an appropriate discount rate) above $\$ 250 \times 0.2=\$ 50$ per Ampere-hour of capacity fade will correspond to an acceptable investment in datacenter demand response capabilities.

\subsection{Optimal parameters and robustness of the PI controller}

This section intends to explore the performance of the PI controller to achieve the demand response objectives in comparison to the P controller. The Pareto fronts in Figure 6.a show very little improvement in optimization objectives from the P controller to the PI controller. The reason behind that lies in how the objective functions are defined. Cost savings are direct functions of peak power reduction. Moreover, large peak reduction requires large SOC swing and high current through each cell which accelerates battery aging. Therefore, in most of the cases, minimizing the steady-state error in SOC tracking does not improve the objective functions significantly. As a result, the optimal values of $\alpha, \beta$ and $S O C_{d e s}$ for PI controller shown in Figure 6.b to 6.d follow similar trends as the P controller. In addition, most of the optimal PI controllers have small integral gains $(\delta)$ shown in Figure 6.e. One minor difference between the Pareto fronts for the P and PI controllers is visible at the leftmost edges of these fronts. At this edge, 
the P controllers prioritize battery health over peak shaving by using very small values of the feedforward gain $\alpha$. In contrast, the PI controllers are able to track the desired SOC more accurately, thanks to integral feedback. This allows the PI controllers to use slightly higher feedforward gains, thereby achieving more cost savings for the same levels of degradation. This is a minor effect, visible only over a small portion of the Pareto front.

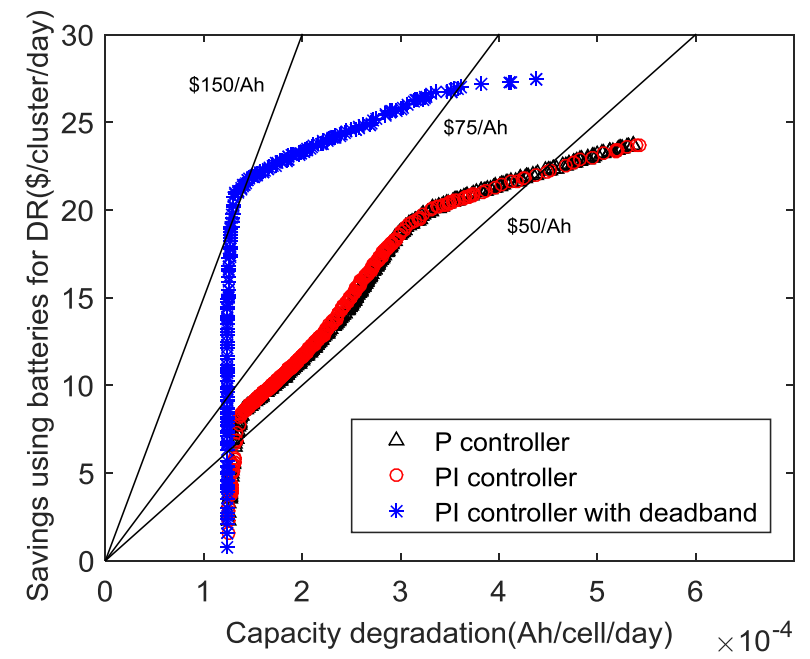

(a)

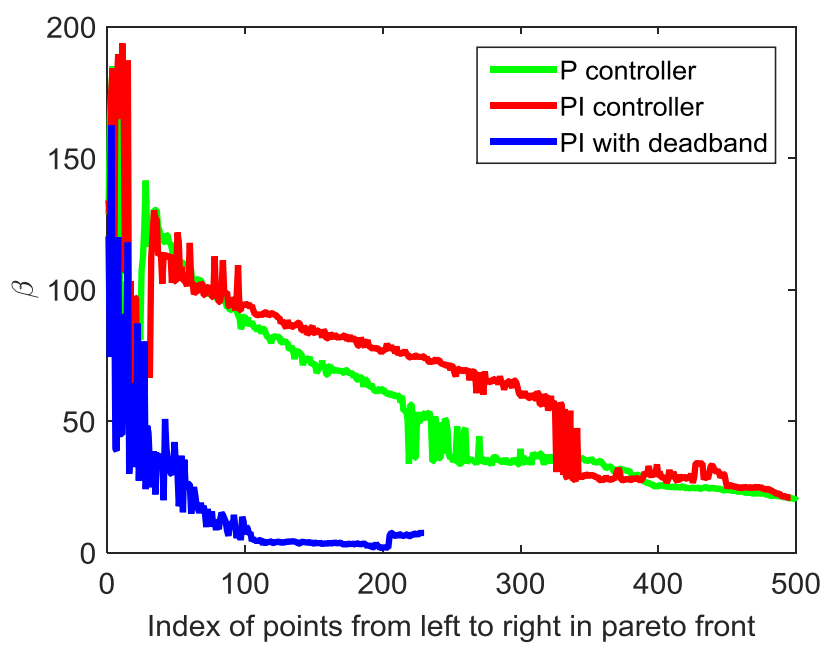

(c)

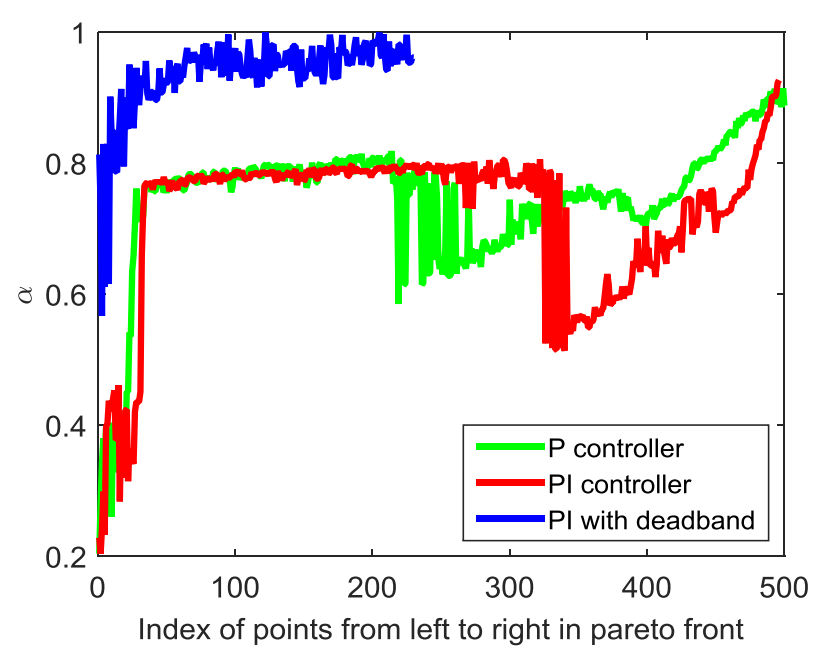

(b)

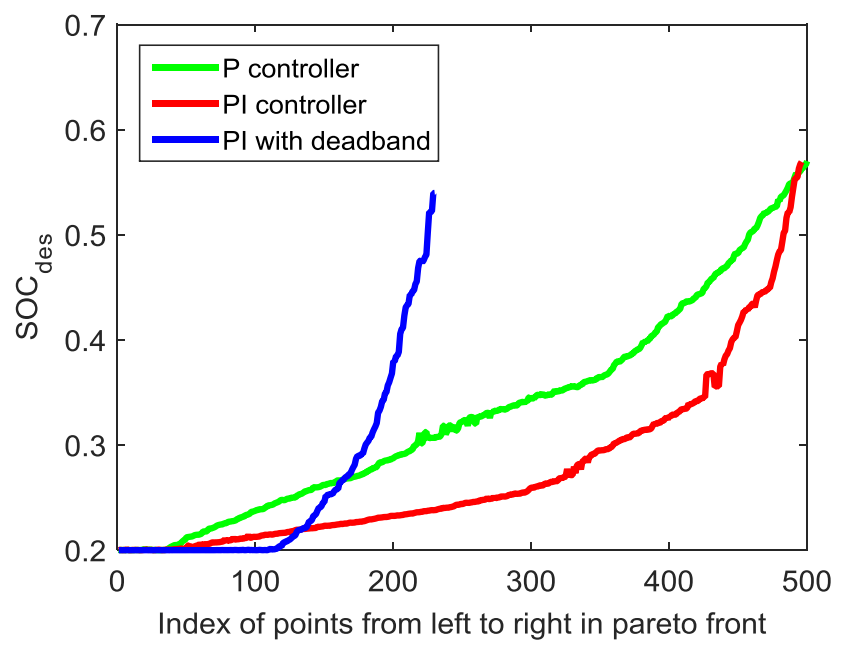

(d) 


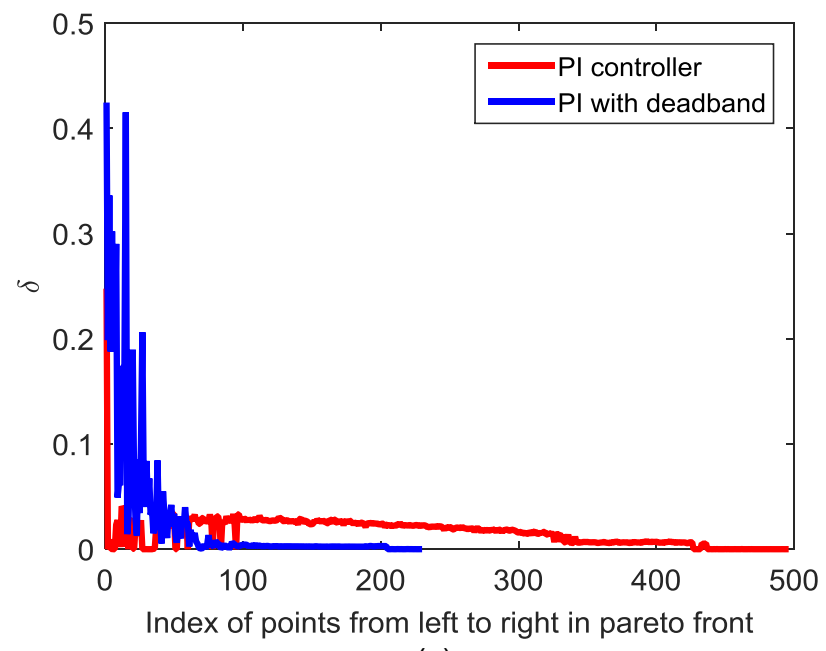

(e)

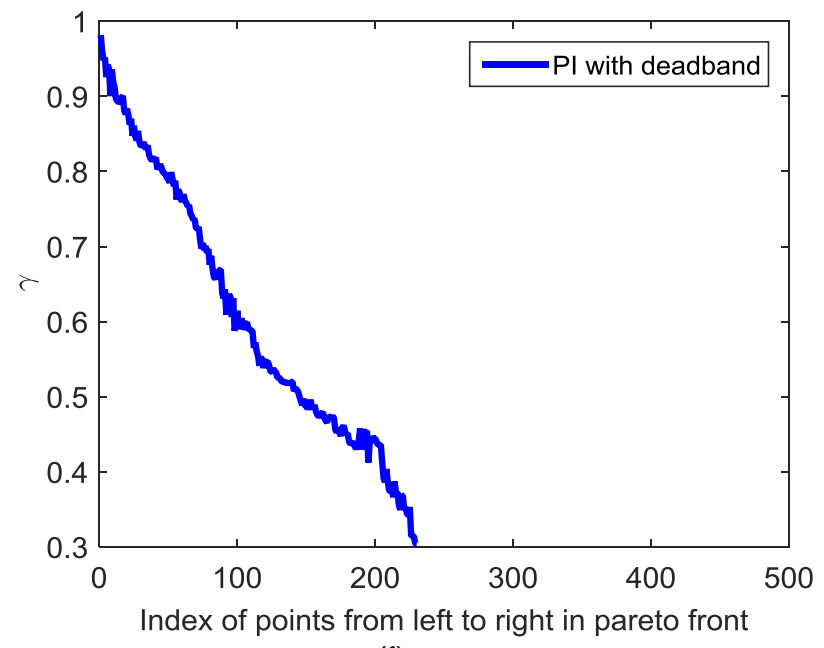

(f)

Fig. 6. a) Pareto front for a battery pack with 1000 cells; Variations of b) $\alpha$; c) $\beta$; d) $S O C_{d e s}$; e) $\delta$; f) $\gamma$ along the Pareto front. (Double column fitting image)

Even though the PI controller does not improve the optimization objectives significantly, it increases the robustness of the control design. To illustrate the idea, a P and a PI controller design that produce similar results in terms of the optimization objectives are selected from their respective fronts. Datacenter demand response is then simulated with these two optimal controller designs for a demand profile which is shifted down by $10 \mathrm{~W}$ compared to the expected demand profile. However, the shifted demand profile is not an actual datacenter demand profile, rather generated for illustration purpose by subtracting $10 \mathrm{~W}$ from the baseline demand profile used in this work. Figure 7 shows that for the new power demand profile, the P controller results in a shifted SOC curve where the PI controller can still track the desired SOC. Figure 8 shows the controller performance in terms of the objective functions. Even after shifting the power demand, the PI controller can achieve roughly the same amount of capacity fade and savings. In contrast, for the $\mathrm{P}$ controller, capacity fade increases nearly $50 \%$ with minor increase in savings. Therefore, when the datacenter power demand deviates from the expected demand profile, the PI controller with a large integral gain performs better then P controller in tracking the desired SOC and can maintain the optimality of the demand response objectives. This is consistent with the established fact from feedback control theory that integral feedback is capable of handling uncertainties in power demand, also known as "disturbances", more robustly than proportional control. 

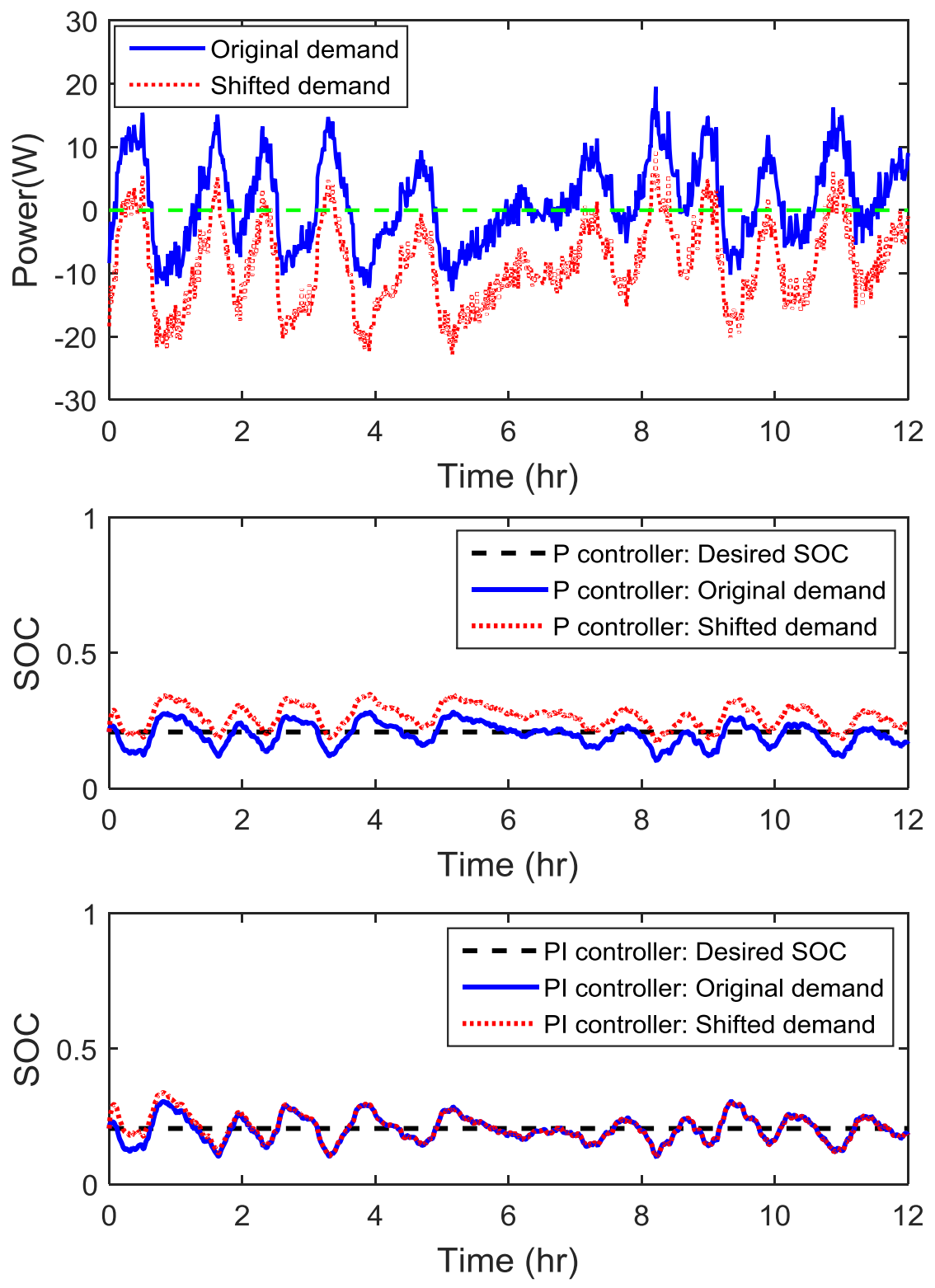

Fig. 7. Performance of the PI controller compared to P controller in SOC tracking when power demand deviates from the expected demand. (Single column fitting image) 


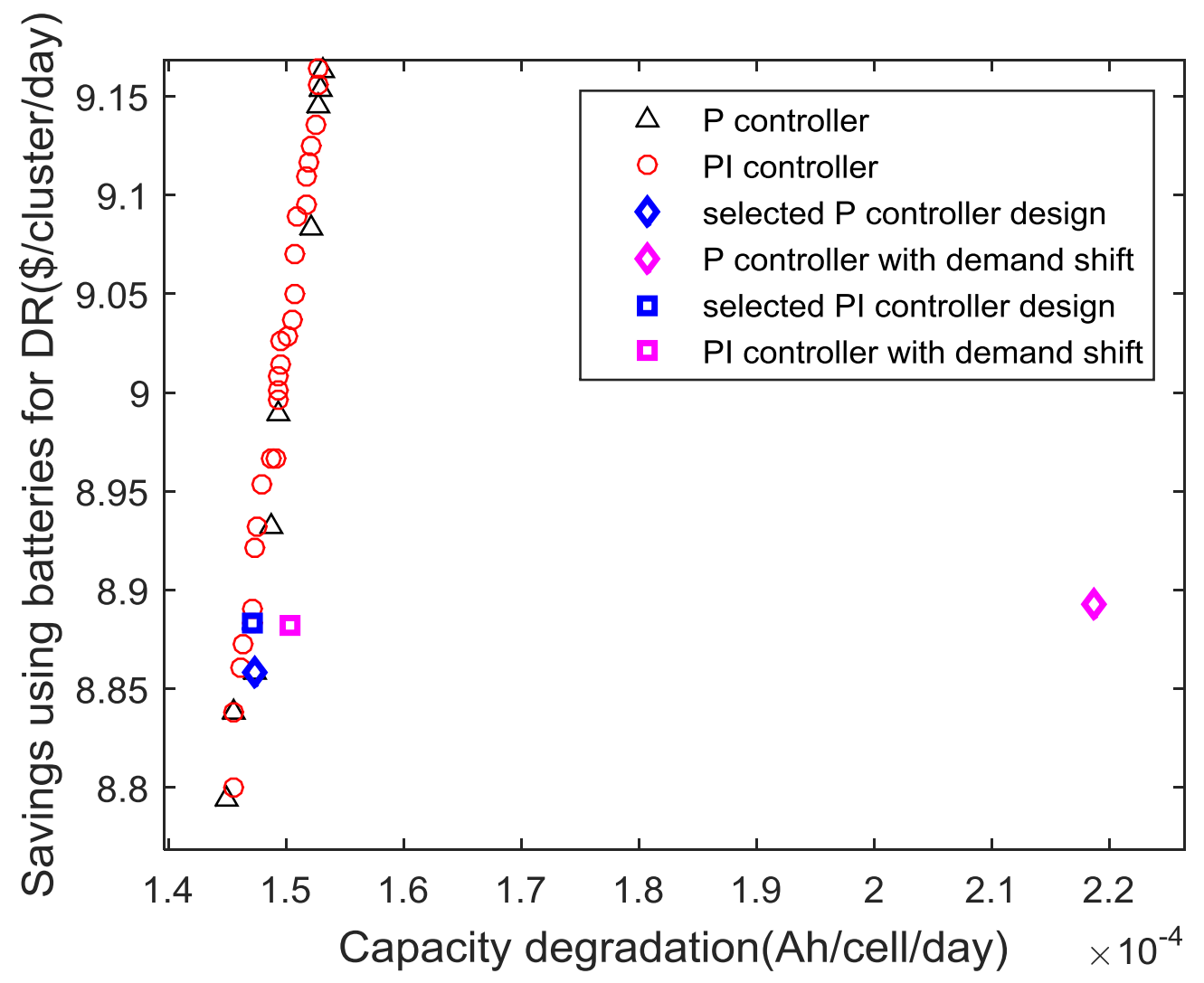

Fig. 8. Change in objective function values from optimal for $\mathrm{P}$ and PI controller due to change in power demand from the expected demand. (Single column fitting image)

\subsection{Performance of the PI controller with a deadband}

This section explains the improvements in optimization objectives in comparison to other two controller variations when a deadband is introduced in the feedforward component of the PI controller. The deadband in this article represents a threshold power demand to start peak shaving and valley filling. Figure 6.a shows significant improvement of the objective functions when a deadband is introduced in the feedforward component of the PI controller. Adding the width of the deadband as an optimization variable allows the optimizer to explore an extended region of the objective function space. In this region, non-zero values of $\gamma$ (Figure 6.f) ensure no peak shaving and valley filling when power demand is within the deadband. In addition to that, large average values of $\alpha$ (Figure 6.b) allow the controller to shave power peaks outside the deadband more effectively compared to other controllers. As we traverse from 
left to right in the Pareto front, the width of the deadband reduces and more savings are obtained since the controller effectively shaves smaller peaks as well.

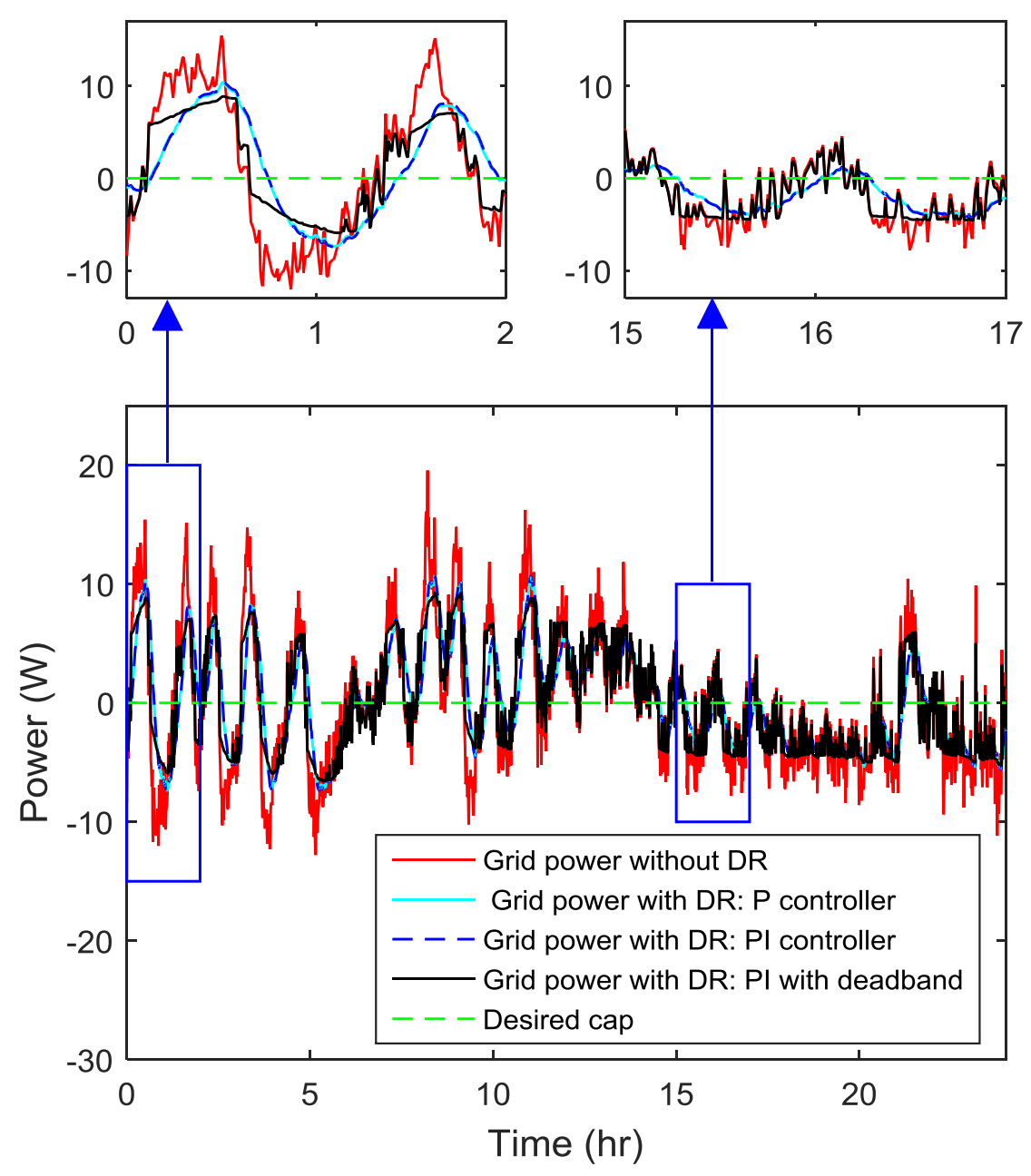

Fig. 9. Performance of the controllers to shave power peaks with maximum emphasis on dollar savings and minimum emphasis on degradation. (Single column fitting image)

To demonstrate how these three controllers perform in terms of peak shaving during demand response, the rightmost point of each Pareto front is selected. In these points each controller puts most emphasis on savings and least emphasis on degradation. Figure 9 shows the desired power cap and the demand smoothing obtained when batteries with each controller are used for demand response. The P and PI controller perform similarly to smooth out fluctuations irrespective of the peak height. In contrast, the 
PI controller with deadband reacts only when power demand deviates significantly from the desired cap. For smaller peaks the battery remains unused since shaving smaller peaks does not provide substantial economic benefit. This controller also performs better smoothing compared to the P and PI controller when power demand is very high. Therefore, adding a deadband in the feedforward component of the PI controller significantly improves the ability of the controller to achieve minimum cost and battery aging during demand response.

\subsection{Impact of battery pack size on controller design}

The above optimization framework is also useful for sizing the Lithium-ion battery pack for demand response. Figure 10 shows the Pareto fronts obtained by implementing the P controller and the PI controller with deadband for battery pack with 1000, 1500, and 2000 cells. For a larger battery pack size, the Pareto fronts move to the left where battery degradation per cell is smaller. The reason for this shift is the decrease in power drawn per cell compared to a smaller battery pack. Due to large power drawn per cell in a smaller battery pack, it is more difficult for the optimizer to find a set of control parameters that can keep the cell within its physical limits. The amount of savings per day also increases for a bigger battery pack, since better load leveling is possible given the same range of physical limits. Therefore, higher initial investments in battery storage will result in a longer battery lifetime and larger savings. Please note that the "savings" plotted in these Pareto fronts represent capital and operating expense reductions associated with the datacenter's electricity infrastructure and electricity consumption, respectively. The capital cost associated with the acquisition of the batteries are not accounted for in these plots, in order to keep all Pareto plots in this mutually consistent. Figure 10 also shows that the PI controller with deadband is more robust to change in battery pack size compared to $\mathrm{P}$ controller in terms of the optimization objectives. The reason behind that is the ability of the PI controller with deadband to utilize the cell capacity more efficiently which leaves less room for further improvement of the optimization objectives. 


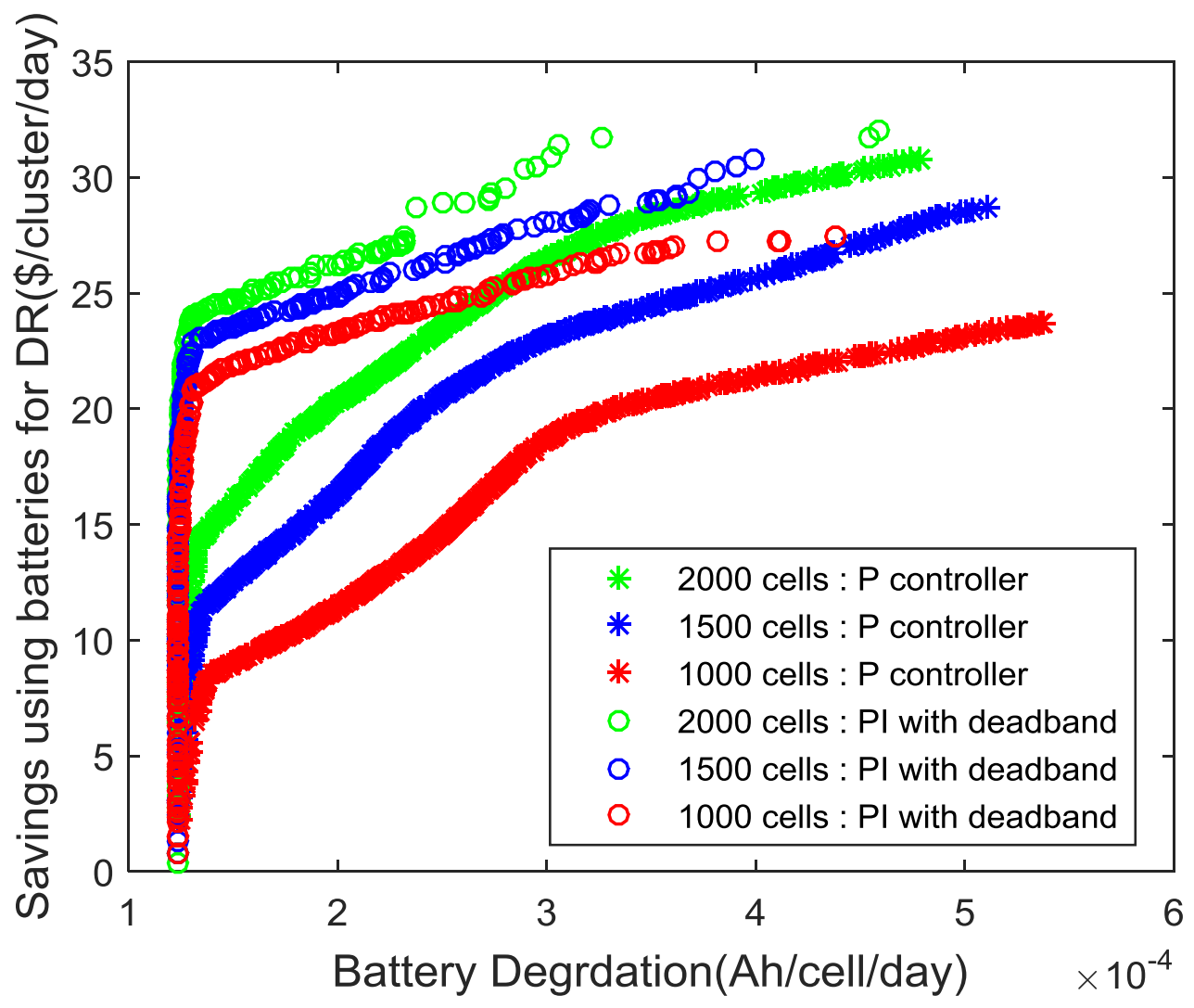

Fig. 10. Comparison among Pareto fronts for different battery pack sizes. (Single column fitting image)

\section{Conclusions}

This article develops a multi-objective optimization framework for datacenter demand response using a Lithium-ion battery pack. We optimize and compare different versions of a feedforward-feedback control strategy to achieve minimum degradation and maximum economic savings. A nonlinear equivalent circuit model and SEI layer growth-based aging model are used to capture battery dynamics and degradation, respectively. The lessons learned from this body of work can be summarized as follows:

- There are significant tradeoffs between dollar savings and battery aging when Lithium-ion batteries are used for demand response in datacenters. These tradeoffs furnish a Pareto front when the optimizer tries to achieve both objectives at the same time using a battery controller.

- Controller gains and parameters follow specific trends to have different levels of emphasis on battery degradation and electricity cost when a proportional feedforward-feedback battery controller is used. 
- Adding an integrator in the feedback loop and optimizing for unknown controller parameters does not result in notable improvement of the objectives. However, the optimal PI controller with high integral gain ensures robustness of the control design and maintains optimality when power demand deviates from the expected behavior.

- Introducing a deadband in the feedforward component and optimizing the controller parameters for selective peak shaving and valley filling improves the optimization objectives significantly. It moves the Pareto front towards higher savings and lower battery aging compared to the other two controller design. Hence it is the better option for health-conscious optimal demand response in datacenters.

- The Pareto front of competing objectives shift toward higher savings and smaller health degradation for larger battery pack sizes. The PI controller with deadband is more robust to change in battery pack size compared to the $\mathrm{P}$ controller in terms of the optimization objectives

\section{Acknowledgments}

This work is supported by National Science Foundation grant CNS-1302225, "CSR: medium: Provisioning and Harnessing Energy Storage for Datacenter Demand Response". The authors gratefully acknowledge this support. The authors would also like to thank Kushagra Vaid from Cloud Server Infrastructure team in Microsoft for providing power traces for validating the battery model.

\section{Nomenclature}

$\begin{array}{ll}A_{n} & \text { Sheet area of negative electrode }\left(\mathrm{m}^{2}\right) \\ C_{\text {cap }} & \text { Capital cost of power provisioning }\left(\$ \mathrm{~W}^{-1}(12 \text { years })^{-1}\right) \\ C_{e} & \text { Concentration of lithium-ion in the electrolyte }\left(\mathrm{mol} \mathrm{m}{ }^{-3}\right) \\ C_{\text {energy }} & \text { Unit cost of energy }\left(\$(\mathrm{kWh})^{-1}\right) \\ C_{\text {peak }} & \text { Unit cost of peak power }\left(\$ \mathrm{~kW}^{-1} \mathrm{month}^{-1}\right)\end{array}$




\begin{tabular}{|c|c|}
\hline$C_{R}$ & Charge capacity of the capacitor in the RC pair $(\mathrm{F})$ \\
\hline$C_{s, n, \max }$ & Maximum concentration of lithium-ion in the negative electrode $\left(\mathrm{mol} \mathrm{m}^{-3}\right)$ \\
\hline$C_{s, n, \text { surf }}$ & Surface concentration of lithium-ion in the negative electrode $\left(\mathrm{mol} \mathrm{m}^{-3}\right)$ \\
\hline$E_{\text {charge }}$ & Energy stored in the battery (Wh) \\
\hline$E_{\text {discharge }}$ & Energy released from the battery $(\mathrm{Wh})$ \\
\hline$F$ & Faraday's constant $\left(\mathrm{C} \mathrm{mol}^{-1}\right)$ \\
\hline$I$ & Input Current (A) \\
\hline$J_{\text {main }}$ & Volumetric current density of the main reaction $\left(\mathrm{A} \mathrm{m}^{-3}\right)$ \\
\hline$J_{\text {side }}$ & Volumetric current density of the side reaction $\left(\mathrm{A} \mathrm{m}^{-3}\right)$ \\
\hline$J_{\text {tot }}$ & Total intercalation current $\left(\mathrm{A} \mathrm{m}^{-3}\right)$ \\
\hline$L_{n}$ & Thickness of the negative electrode (m) \\
\hline$M_{p}$ & Molar mass of SEI layer material $\left(\mathrm{kg} \mathrm{mol}^{-1}\right)$ \\
\hline$P_{\text {batt }}$ & Battery input power $(\mathrm{W})$ \\
\hline$P_{\text {batt }, \text { dis }}$ & Battery discharge power $(\mathrm{W})$ \\
\hline$P_{\text {cap,des }}$ & Desired power cap for "ideal" load leveling (W) \\
\hline$P_{\text {cap, } \max }$ & Peak power demanded from utilities with demand response $(\mathrm{W})$ \\
\hline$P_{d}$ & Datacenter power demand (W) \\
\hline$P_{d, a v g}$ & Average datacenter power demand (W) \\
\hline$P_{d, \max }$ & Maximum datacenter power demand (W) \\
\hline$P_{d, \min }$ & Minimum datacenter power demand (W) \\
\hline$Q$ & Cell charge capacity $(\mathrm{C})$ \\
\hline$Q_{\text {loss }}$ & Capacity lost due to side reaction (Ah) \\
\hline$R$ & Lumped resistance of the cell and the connector $(\Omega)$ \\
\hline$R_{\text {film }}$ & Total film resistance $\left(\Omega \mathrm{m}^{-2}\right)$ \\
\hline
\end{tabular}




\begin{tabular}{|c|c|}
\hline$R_{r}$ & Resistance of the resistor in the RC pair $(\Omega)$ \\
\hline$R_{S E I}$ & Initial resistance of SEI layer $\left(\Omega \mathrm{m}^{-2}\right)$ \\
\hline$R_{u}$ & Universal gas constant $\left(\mathrm{J} \mathrm{mol}^{-1}\right)$ \\
\hline$S O C_{d e s}$ & Desired SOC of the cell \\
\hline$T$ & Cell temperature $(\mathrm{K})$ \\
\hline$U_{n, r e f}$ & Main reaction equilibrium potential of the negative electrode $(\mathrm{V})$ \\
\hline$U_{s, r e f}$ & Side reaction equilibrium potential of $(\mathrm{V})$ \\
\hline$V$ & Terminal voltage $(\mathrm{V})$ \\
\hline$V_{o c}$ & Open circuit voltage (V) \\
\hline$X_{1}$ & Cell state of charge \\
\hline$X_{2}$ & Charge in the capacitor in the $\mathrm{RC}$ pair $(\mathrm{C})$ \\
\hline$Z$ & Optimization objective \\
\hline$a_{n}$ & Specific surface area of the negative electrode $\left(\mathrm{m}^{-1}\right)$ \\
\hline$i_{o, n}$ & Main reaction exchange current density in the negative electrode $\left(\mathrm{A} \mathrm{m}^{-2}\right)$ \\
\hline$i_{o, s}$ & Side reaction exchange current density $\left(\mathrm{A} \mathrm{m}^{-2}\right)$ \\
\hline$k_{n}$ & Reaction rate constant at the negative electrode $\left(\mathrm{A} \mathrm{m}^{-2}\left(\mathrm{~mol} \mathrm{~m}^{-3}\right)^{1.5}\right)$ \\
\hline$x_{n, \text { surf }}$ & Surface state of charge of the negative electrode \\
\hline$\Delta \varphi$ & Potential difference between solid and electrolyte (V) \\
\hline$\alpha, \beta$ & Proportional gain \\
\hline$\alpha_{a}, \alpha_{c}$ & Apparent charge transfer coefficients for anode and cathode \\
\hline$\gamma$ & Dead band width gain \\
\hline$\delta$ & Integral gain \\
\hline$\delta_{\text {film }}$ & Time varying SEI film thickness (m) \\
\hline$\eta_{n}$ & Main reaction overpotential at the negative electrode $(\mathrm{V})$ \\
\hline
\end{tabular}




$\begin{array}{ll}\eta_{s} & \text { Side reaction overpotential }(\mathrm{V}) \\ \kappa_{P} & \text { Conductivity of the SEI film } \\ \rho_{p} & \text { Density of side reaction product }\left(\mathrm{kg} \mathrm{m}^{-3}\right) \\ \tau & \text { Time constant (s) }\end{array}$

\section{References}

[1] R. Urgaonkar, B. Urgaonkar, M.J. Neely, A. Sivasubramaniam, Optimal power cost management using stored energy in data centers, in: Proc. ACM SIGMETRICS Jt. Int. Conf. Meas. Model. Comput. Syst. - SIGMETRICS '11, ACM Press, New York, New York, USA, 2011: p. 221. doi:10.1145/1993744.1993766.

[2] L. Ganesh, J. Liu, N. Suman, F. Zhao, Unleash Stranded Power in Data Centers with RackPacker, in: Work. Energy-Efficient Des., Austin, Texas, 2009. http://research.microsoft.com/apps/pubs/default.aspx?id=80953 (accessed May 10, 2014).

[3] A. Vasan, A. Sivasubramaniam, V. Shimpi, T. Sivabalan, R. Subbiah, Worth their watts? an empirical study of datacenter servers, in: HPCA - 162010 Sixt. Int. Symp. HighPerformance Comput. Archit., IEEE, 2010: pp. 1-10. doi:10.1109/HPCA.2010.5463056.

[4] S. Govindan, A. Sivasubramaniam, B. Urgaonkar, Benefits and limitations of tapping into stored energy for datacenters, in: Proc. 38th Annu. Int. Symp. Comput. Archit. (ISCA '11), ACM, New York, NY, USA, 2011: pp. 341-352. doi:10.1145/2024723.2000105.

[5] S. Govindan, D. Wang, A. Sivasubramaniam, B. Urgaonkar, Leveraging stored energy for handling power emergencies in aggressively provisioned datacenters, in: Proc. Seventeenth Int. Conf. Archit. Support Program. Lang. Oper. Syst. (ASPLOS XVII), New York, NY, USA., 2012: pp. 75-86. doi:10.1145/2189750.2150985.

[6] D. Wang, C. Ren, S. Govindan, A. Sivasubramaniam, B. Urgaonkar, A. Kansal, et al., ACE: Abstracting, characterizing and exploiting datacenter power demands, 2013 IEEE Int. Symp. Workload Charact. (2013) 44-55. doi:10.1109/IISWC.2013.6704669.

[7] C. Lefurgy, X. Wang, M. Ware, Server-Level Power Control, in: Fourth Int. Conf. Auton. Comput., IEEE, 2007: pp. 4-4. doi:10.1109/ICAC.2007.35.

[8] S. Govindan, J. Choi, B. Urgaonkar, A. Sivasubramaniam, A. Baldini, Statistical profilingbased techniques for effective power provisioning in data centers, in: Proc. Fourth ACM Eur. Conf. Comput. Syst. - EuroSys '09, ACM Press, New York, New York, USA, 2009: p. 317. doi:10.1145/1519065.1519099.

[9] X. Wang, M. Chen, Cluster-level feedback power control for performance optimization, in: 2008 IEEE 14th Int. Symp. High Perform. Comput. Archit., IEEE, 2008: pp. 101-110. doi:10.1109/HPCA.2008.4658631.

[10] X. Fan, W.-D. Weber, L.A. Barroso, Power provisioning for a warehouse-sized computer, ACM SIGARCH Comput. Archit. News. 35 (2007) 13. doi:10.1145/1273440.1250665.

[11] E. Pakbaznia, M. Pedram, Minimizing data center cooling and server power costs, in: Proc. 14th ACM/IEEE Int. Symp. Low Power Electron. Des. - ISLPED '09, ACM Press, New York, New York, USA, 2009: p. 145. doi:10.1145/1594233.1594268.

[12] T.D. Boucher, D.M. Auslander, C.E. Bash, C.C. Federspiel, C.D. Patel, Viability of 
Dynamic Cooling Control in a Data Center Environment, J. Electron. Packag. 128 (2006) 137. doi:10.1115/1.2165214.

[13] E. Pakbaznia, M. Ghasemazar, M. Pedram, Temperature-aware dynamic resource provisioning in a power-optimized datacenter, (2010) 124-129. http://dl.acm.org/citation.cfm?id=1870926.1870959 (accessed December 7, 2014).

[14] A. Mamun, D. Wang, I. Narayanan, A. Sivasubramaniam, H.K. Fathy, Physics-based simulation of the impact of demand response on lead-acid emergency power availability in a datacenter, J. Power Sources. 275 (2015) 516-524. doi:10.1016/j.jpowsour.2014.11.025.

[15] D. Wang, S. Govindan, A. Sivasubramaniam, A. Kansal, J. Liu, B. Khessib, Underprovisioning backup power infrastructure for datacenters, in: Proc. 19th Int. Conf. Archit. Support Program. Lang. Oper. Syst. - ASPLOS '14, ACM Press, New York, New York, USA, 2014: pp. 177-192. doi:10.1145/2541940.2541966.

[16] V. Kontorinis, L.E. Zhang, B. Aksanli, J. Sampson, H. Homayoun, E. Pettis, et al., Managing distributed ups energy for effective power capping in data centers, ACM SIGARCH Comput. Archit. News. 40 (2012) 488. doi:10.1145/2366231.2337216.

[17] L.A. Barroso, U. Hölzle, The Datacenter as a Computer: An Introduction to the Design of Warehouse-Scale Machines, Synth. Lect. Comput. Archit. 4 (2009) 1-108. doi:10.2200/S00193ED1V01Y200905CAC006.

[18] Y. Guo, Z. Ding, Y. Fang, D. Wu, Cutting Down Electricity Cost in Internet Data Centers by Using Energy Storage, in: 2011 IEEE Glob. Telecommun. Conf. - GLOBECOM 2011, Ieee, 2011: pp. 1-5. doi:10.1109/GLOCOM.2011.6134209.

[19] D. Wang, C. Ren, A. Sivasubramaniam, B. Urgaonkar, H. Fathy, Energy storage in datacenters, in: Proc. 12th ACM SIGMETRICS/PERFORMANCE Jt. Int. Conf. Meas. Model. Comput. Syst. - SIGMETRICS '12, ACM Press, New York, New York, USA, 2012: p. 187. doi:10.1145/2254756.2254780.

[20] B. Aksanli, T. Rosing, E. Pettis, Distributed battery control for peak power shaving in datacenters, 2013 Int. Green Comput. Conf. Proceedings, IGCC 2013. (2013). doi:10.1109/IGCC.2013.6604477.

[21] C. Ren, D. Wang, B. Urgaonkar, A. Sivasubramaniam, Carbon-Aware Energy Capacity Planning for Datacenters, in: 2012 IEEE 20th Int. Symp. Model. Anal. Simul. Comput. Telecommun. Syst., IEEE, 2012: pp. 391-400. doi:10.1109/MASCOTS.2012.51.

[22] A. Mamun, I. Narayanan, D. Wang, A. Sivasubramaniam, H.K. Fathy, Multi-objective Optimization to Minimize Battery Degradation and Electricity Cost for Demand Response in Datacenters, in: ASME 2015 Dyn. Syst. Control Conf., 2015.

[23] I. Narayanan, D. Wang, A. Mamun, A. Sivasubramaniam, H.K. Fathy, Should we DualPurpose Energy Storage in Datacenters for Power Backup and Demand Response ?, in: 6th Work. Power-Aware Comput. Syst., Broomfield,CO, 2014: pp. 1-5.

[24] D. Docimo, M. Ghanaatpishe, H.K. Fathy, Development and Experimental Parameterization of a Physics-Based Second-Order Lithium-Ion Battery Model, in: ASME 2014 Dyn. Syst. Control Conf., 2014: pp. V001T19A003-V001T19A003.

[25] P. Ramadass, B. Haran, P.M. Gomadam, R. White, B.N. Popov, Development of First Principles Capacity Fade Model for Li-Ion Cells, J. Electrochem. Soc. 151 (2004) A196. doi:10.1149/1.1634273.

[26] S.J. Moura, J.C. Forman, S. Bashash, J.L. Stein, H.K. Fathy, Optimal control of film growth in lithium-ion battery packs via relay switches, IEEE Trans. Ind. Electron. 58 (2011) 3555-3566. doi:10.1109/TIE.2010.2087294. 
[27] M. Safari, C. Delacourt, Modeling of a Commercial Graphite/LiFePO4 Cell, J. Electrochem. Soc. 158 (2011) A562-A571. doi:10.1149/1.3567007.

[28] T.R. Tanim, C.D. Rahn, Aging formula for lithium ion batteries with solid electrolyte interphase layer growth, J. Power Sources. 294 (2015) 239-247. doi:10.1016/j.jpowsour.2015.06.014.

[29] J. Liu, G. Li, H.K. Fathy, A Computationally Efficient Approach for Optimizing LithiumIon Battery Charging, J. Dyn. Syst. Meas. Control. 138 (2015) 021009. doi:10.1115/1.4032066.

[30] N. Energy, Explanation of Demand Charge, (n.d.). https://www.northwesternenergy.com/docs/default-source/documents/E-Programs/Edemandcharges.pdf (accessed January 1, 2015).

[31] R. Storn, K. Price, Differential evolution-a simple and efficient heuristic for global optimization over continuous spaces, J. Glob. Optim. 11 (1997) 341-359. http://www.springerlink.com/index/x555692233083677.pdf (accessed March 18, 2013). 